Bull. Austral. Math. Soc.

VoL. 58 (1998) [33-41]

\title{
FIXED POINT THEOREMS AND EQUILIBRIUM POINTS IN ABSTRACT ECONOMIES
}

\author{
DONAL O'REgan
}

New fixed point theorems are given which have applications in the theory of abstract economies.

\section{INTRODUCTION}

The purpose of this paper is twofold. First we present new fixed point theorems for DKT maps. These will then be used to establish new criteria for the existence of an equilibrium point for an abstract economy. The results in this paper improve and complement those in $[\mathbf{2}, \mathbf{8}, \mathbf{9}]$.

Let $Z$ be a subset of a Hausdorff topological space $E_{1}$ and $W$ a subset of a topological space $E_{2}$. We say $F \in D K T(Z, W)$ if $W$ is convex, and there exists a map $B: Z \rightarrow W$ with $c o(B(x)) \subseteq F(x)$ for all $x \in Z, B(x) \neq \emptyset$ for each $x \in Z$ and the fibres $B^{-1}(y)$ are open (in $Z$ ) for each $y \in W$. The following selection theorem will be used in Section 2 (see $[2$, p.206] for a proof).

THEOREM 1.1. Let $Z$ be a nonempty, paracompact Hausdorff topological space and $W$ a nonempty, convex subset of a topological vector space. Suppose $F \in$ $D K T(Z, W)$. Then $F$ has a continuous selection (that is, there exists a continuous single valued map $f: Z \rightarrow W$ of $F$ ).

\section{FiXed POINT THEORY AND APPLICATIONS}

We begin by establishing some new fixed point results for $D K T$ maps. Our analysis will rely on Theorem 1.1 and on well known fixed point results in the literature (see [3]). The first result concerns compact maps (see [2]).

THEOREM 2.1. Let $I$ be an index set and $\left\{Q_{i}\right\}_{i \in I}$ a family of nonempty, convex sets each in a locally convex Hausdorff linear topological space $E_{i}$. For each $i \in I$, let $G_{i} \in D K T\left(Q, Q_{i}\right)$ be a compact map; here $Q=\prod_{i \in I} Q_{i}$. Then there exists $x^{\star} \in Q$ with $x^{\star} \in G\left(x^{\star}\right) \equiv \prod_{i \in I} G_{i}\left(x^{\star}\right)$ that is, $x_{i}^{\star} \in G_{i}\left(x^{\star}\right)$ for all $i \in I$ (here $x_{i}^{\star}$ is the projection of $x^{\star}$ on $E_{i}$ ).

Received 17th November, 1997

Copyright Clearance Centre, Inc. Serial-fee code: 0004-9729/98 \$A2.00+0.00. 
Proof: For each $i \in I$, choose a compact set $X_{i} \subseteq E_{i}$ such that $G_{i}(Q) \subseteq X_{i} \subseteq Q_{i}$. Let $X=\prod_{i \in I} X_{i}$. Note $X$ is compact. Let $Y=c o(X)$ and notice $[2, \mathrm{p} .206]$ that $Y$ is paracompact. Also it is easy to see that $G_{i} \in D K T\left(Y, \operatorname{co}\left(X_{i}\right)\right)$ for each $i \in I$ (note $G_{i}(Y) \subseteq G_{i}(Q) \subseteq X_{i} \subseteq c o\left(X_{i}\right)$ ). By Theorem 1.1, $G_{i}$ has a continuous selection $g_{i}: Y \rightarrow c o\left(X_{i}\right)$ for each $i \in I$ (in fact $g_{i}: Y \rightarrow X_{i}$ since $\left.g_{i}(Y) \subseteq G_{i}(Y) \subseteq X_{i}\right)$. Let $g: Y \rightarrow Y$ be defined by

$$
g(x)=\prod_{i \in I} g_{i}(x)
$$

note $g(Y) \subseteq \prod_{i \in I} X_{i}=X \subseteq Y$. Also $g$ is continuous and compact since $g(Y) \subseteq X$. Now the Schauder-Tychonoff Theorem [3] implies that there exists $x^{\star} \in Y \subseteq Q$ such that

$$
x^{\star}=g\left(x^{\star}\right)=\prod_{i \in I} g_{i}\left(x^{\star}\right) \subseteq \prod_{i \in I} G_{i}\left(x^{\star}\right) .
$$

Next we improve Theorem 2.1 when our spaces $E_{i}$ are Banach (in fact Theorem 2.2 can easily be extended to the Fréchet space setting).

THEOREM 2.2. Let $I$ be a countable index set and $\left\{Q_{i}\right\}_{i \in I}$ a family of nonempty, convex sets each in a Banach space $E_{i}$. For each $i \in I$, let $G_{i} \in D K T\left(Q, E_{i}\right)$ with $Q=\prod_{i \in I} Q_{i}$. Define $G: Q \rightarrow 2^{E}$ (here $E=\prod_{i \in I} E_{i}$ and note $2^{E}$ denotes the family of nonempty subsets of $E$ ) by

$$
G(x)=\prod_{i \in I} G_{i}(x) \text { for each } x \in Q .
$$

Suppose $G: Q \rightarrow 2^{Q}$ is a condensing map with $G(Q)$ a subset of a bounded set in $Q$. Then there exists $x^{\star} \in Q$ with $x^{\star} \in G\left(x^{\star}\right)$.

Proof: Let $x_{0} \in Q$. Note since $Q$ is closed and convex then $[4, \mathrm{p} .19]$ implies that there exists a closed, convex set $X \subseteq E=\prod_{i \in I} E_{i}$ with $x_{0} \in X$ and

$$
X=\overline{c o}\left(G(Q \cap X) \cup\left\{x_{0}\right\}\right) .
$$

Now since $G(Q \cap X) \cup\left\{x_{0}\right\} \subseteq G(Q) \cup\left\{x_{0}\right\} \subseteq Q$ we have $X \subseteq Q$ and so $Q \cap X=X$. Thus

$$
X=\overline{c o}\left(G(X) \cup\left\{x_{0}\right\}\right) .
$$

Since $G$ is condensing we have, using the properties of measure of noncompactness, that $X$ is compact. Thus $G: X \rightarrow X$ with $X$ convex and compact.

Also $G_{i} \in D K T\left(X, Q_{i}\right)$ together with Theorem 1.1 (note $X$ is paracompact) implies that $G_{i}$ has a continuous selection $g_{i}: X \rightarrow Q_{i}$. Define the map $g: X \rightarrow Q$ by

$$
g(x)=\prod_{i \in I} g_{i}(x) \text { for each } x \in X
$$


Notice $g: X \rightarrow X$ since if $x \in X$ then

$$
g(x) \subseteq \prod_{i \in I} G_{i}(x)=G(x) \subseteq X
$$

Thus $g: X \rightarrow X$ is continuous with $X$ a convex, compact set. Then there exists $x^{\star} \in X \subseteq Q$ with $x^{\star}=g\left(x^{\star}\right) \in G\left(x^{\star}\right)$.

Our next fixed point result improves Theorem 2.1 if our spaces $E_{i}$ are metrisable.

THEOREM 2.3. Let $I$ be a countable index set and $\left\{Q_{i}\right\}_{i \in I}$ a family of nonempty, closed, convex sets each in a metrisable locally convex linear topological space $E_{i}$. Let $Q=\prod_{i \in I} Q_{i}$ and suppose $0 \in Q$. For each $i \in I$, let $G_{i} \in D K T\left(Q, E_{i}\right)$ be a compact map. Define $G: Q \rightarrow 2^{E}$ (here $E=\prod_{i \in I} E_{i}$ ) by

$$
G(x)=\prod_{i \in I} G_{i}(x) \text { for each } x \in Q
$$

Suppose the following condition is satisfied:

$$
\left\{\begin{array}{l}
\text { if }\left\{\left(x_{j}, \lambda_{j}\right)\right\}_{1}^{\infty} \text { is a sequence in } \partial Q \times[0,1] \text { converging to }(x, \lambda) \\
\text { with } x \in \lambda G(x) \text { and } 0 \leqslant \lambda<1, \text { then there exists } j_{0} \in\{1,2, \ldots\} \\
\text { with }\left\{\lambda_{j} G\left(x_{j}\right)\right\} \subseteq Q \text { for each } j \geqslant j_{0} .
\end{array}\right.
$$

Then there exists $x^{\star} \in Q$ with $x^{\star} \in G\left(x^{\star}\right)$.

Proof: Note $Q$ is paracompact since metrisable spaces are paracompact and closed subsets of paracompact spaces are paracompact. For each $i \in I$, by Theorem 1.1, there exists a continuous selection $g_{i}: Q \rightarrow E_{i}$ of $G_{i}$. Define $g: Q \rightarrow E=\prod_{i \in I} E_{i}$ by

$$
g(x)=\prod_{i \in I} g_{i}(x)
$$

Notice $g$ is continuous. Let $r: E \rightarrow Q$ be a continuous retraction with $r(z) \in \partial Q$ for $z \in E \backslash Q$ (see [5]). Consider

$$
X=\{x \in E: x=g r(x)\}
$$

Notice $X \neq \emptyset$; this follows from the Schauder-Tychonoff Theorem since $g r: E \rightarrow E$ is a continuous, compact map (note $g r(E) \subseteq g(Q)=\prod_{i \in I} g_{i}(Q)$ ). Also $X$ is closed and in fact compact since $X \subseteq g r(X) \subseteq g(Q)=\prod_{i \in I} g_{i}(Q)$. It remains to show $X \cap Q \neq \emptyset$. Suppose $X \cap Q=\emptyset$. Then there exists $\delta>0$ with $\operatorname{dist}(X, Q)>\delta$. Choose $m \in\{1,2, \ldots\}$ such that $1<\delta m$. Fix $i \in\{m, m+1, \ldots\}$ and let

$$
U_{i}=\left\{x \in E: d(x, Q)<\frac{1}{i}\right\}
$$


here $d$ is the metric associated with $E$. Then $X \cap \overline{U_{i}}=\emptyset$. Now the nonlinear alternative for compact single valued maps (see [3]) implies (since $X \cap \overline{U_{i}}=\emptyset$ ) that there exists $\left(y_{i}, \lambda_{i}\right) \in \partial U_{i} \times(0,1)$ with $y_{i}=\lambda_{i} g r\left(y_{i}\right)$. Now $\lambda_{i} g r\left(y_{i}\right) \notin Q$ for $i \in\{m, m+1, \ldots\}$ and so

$$
\left\{\lambda_{i} G r\left(y_{i}\right)\right\} \nsubseteq Q \text { for each } i \in\{m, m+1, \ldots\}
$$

Let

$$
D=\{x \in E: x=\lambda g r(x) \text { for some } \lambda \in[0,1]\} \text {. }
$$

Now $D$ is compact (since $D \subseteq \overline{c o}(g(r(D)) \cup\{0\}))$ and so we may assume without loss of generality that $\lambda_{j} \rightarrow \lambda^{\star}$ and $y_{j} \rightarrow y^{\star} \in \partial Q$. Also $y^{\star}=\lambda^{\star} g r\left(y^{\star}\right)$. Now $\lambda^{\star} \neq 1$ since $X \cap Q=\emptyset$. Thus $0 \leqslant \lambda^{\star}<1$. But in this case (2.1), with $x_{j}=r\left(y_{j}\right), x=y^{\star}=r\left(y^{\star}\right)$, implies that there exists $j_{0} \in\{1,2, \ldots\}$ with $\left\{\lambda_{j} G r\left(y_{j}\right)\right\} \subseteq Q$ for each $j \geqslant j_{0}$. This contradicts (2.2). Thus $X \cap Q \neq \emptyset$ that is, there exists $w \in Q$ with $w \in G r(w)=G(w)$. $\square$

In Theorem 2.3 the compactness assumption on the maps $G_{i}$ may be replaced by a condensing assumption on the $\operatorname{map} G$. We follow the reasoning in Theorem 2.3. We need however our spaces $E_{i}$ to be Hilbert spaces; choose the map $r: E \rightarrow Q$ in this case to be the nearest point projection (which we know to be nonexpansive). For completeness we state the result (the minor adjustments in the proof of Theorem 2.3 are left to the reader).

THEOREM 2.4. Let $I$ be a countable index set and $\left\{Q_{i}\right\}_{i \in I}$ a family of nonempty, closed, convex sets each in a Hilbert space $E_{i}$. Let $Q=\prod_{i \in I} Q_{i}$ and suppose $0 \in Q$. For each $i \in I$, let $G_{i} \in D K T\left(Q, E_{i}\right)$. Define $G: Q \rightarrow 2^{E}$ (here $E=\prod_{i \in I} E_{i}$ ) by

$$
G(x)=\prod_{i \in I} G_{i}(x) \text { for each } x \in Q
$$

Suppose $G: Q \rightarrow 2^{E}$ is a condensing map with $G(Q)$ a subset of a bounded set in $E$ and assume (2.1) holds. Then there exists $x^{\star} \in Q$ with $x^{\star} \in G\left(x^{\star}\right)$.

Next we apply Theorem 2.1 and Theorem 2.3 to give equilibrium theorems for an abstract economy. It is worth remarking here that we could also apply Theorem 2.2 and Theorem 2.4 to obtain other equilibrium theorems for an abstract economy. Let $I$ be a countable or uncountable set of agents. We shall describe an abstract economy by $\Gamma=\left(Q_{i}, F_{i}, G_{i}, P_{i}\right)_{i \in I}$ where for each $i \in I, Q_{i}\left(\subseteq E_{i}\right)$ is the choice (or strategy) set, $F_{i}, G_{i}: \prod_{i \in I} Q_{i}=Q \rightarrow 2^{Q_{i}}$ are contraint correspondences and $P_{i}: Q \rightarrow 2^{Q_{i}}$ is a preference correspondence (see $[8,9]$ ). In the case of an abstract economy being given by $\Gamma=\left(Q_{i}, F_{i}, G_{i}, P_{i}\right)_{i \in I}$ a point $x^{\star} \in Q$ is called an equilibrium point for $\Gamma$ (or a generalised Nash equilibrium point) if for each $i \in I, x_{i}^{\star} \in c l_{E_{i}} G_{i}\left(x^{\star}\right)$ and $F_{i}\left(x^{\star}\right) \cap P_{i}\left(x^{\star}\right)=\emptyset$ (here $x_{i}^{\star}$ is the projection of $x^{\star}$ on $E_{i}$ ); if such an $x^{\star}$ exists we say $\Gamma$ has an equilibrium. 
THEOREM 2.5. Let $\Gamma=\left(Q_{i}, F_{i}, G_{i}, P_{i}\right)_{i \in I}$ be an abstract economy such that for each $i \in I$ the following conditions hold:

$$
\left\{\begin{array}{l}
Q_{i} \text { is a nonempty, convex set in a locally convex } \\
\text { Hausdorff linear topological space } E_{i}
\end{array}\right.
$$

$$
\text { for each } x \in Q=\prod_{i \in I} Q_{i}, \quad F_{i}(x) \neq \emptyset \text { and } \operatorname{co}\left(F_{i}(x)\right) \subseteq G_{i}(x)
$$

$$
\left\{\begin{array}{l}
\text { for each } y_{i} \in Q_{i}, \text { the set }\left[\left(\operatorname{co}\left(P_{i}\right)\right)^{-1}\left(y_{i}\right) \cup M_{i}\right] \cap F_{i}^{-1}\left(y_{i}\right) \\
\text { is open in } Q ; \text { here } M_{i}=\left\{x \in Q: F_{i}(x) \cap P_{i}(x)=\emptyset\right\}
\end{array}\right.
$$

$$
G_{i}: Q \rightarrow 2^{Q_{i}} \text { is a compact map }
$$

and

(2.7) for each $x \in Q, x_{i} \notin c o\left(P_{i}(x)\right)$; here $x_{i}$ is the projection of $x$ on $E_{i}$.

Then $\Gamma$ has an equilibrium point $x^{\star} \in Q$, that is, for each $i \in I$,

$$
x_{i}^{\star} \in G_{i}\left(x^{\star}\right) \text { and } F_{i}\left(x^{\star}\right) \cap P_{i}\left(x^{\star}\right)=\emptyset \text {; }
$$

here $x_{i}^{\star}$ is the projection of $x^{\star}$ on $E_{i}$.

Proof: For each $i \in I$ let

$$
N_{i}=\left\{x \in Q: F_{i}(x) \cap P_{i}(x) \neq \emptyset\right\}
$$

and for each $x \in Q$ let

$$
I(x)=\left\{i \in I: F_{i}(x) \cap P_{i}(x) \neq \emptyset\right\} .
$$

For each $i \in I$, define the correspondences $A_{i}, B_{i}: Q \rightarrow 2^{Q_{i}}$ by

$$
A_{i}(x)=\left\{\begin{array}{l}
\left.\operatorname{co} P_{i}(x) \cap F_{i}(x) \text { if } i \in I(x) \text { (that is, } x \in N_{i}\right) \\
F_{i}(x) \text { if } i \notin I(x)
\end{array}\right.
$$

and

$$
B_{i}(x)=\left\{\begin{array}{l}
\operatorname{co} P_{i}(x) \cap G_{i}(x) \text { if } i \in I(x) \\
G_{i}(x) \text { if } i \notin I(x) .
\end{array}\right.
$$

It is easy to see (use (2.4) and the definition of $I(x)$ ) for each $i \in I$ and $x \in Q$ that

$$
\operatorname{co}\left(A_{i}(x)\right) \subseteq B_{i}(x) \text { and } A_{i}(x) \neq \emptyset
$$

Also for each $i \in I$ and $y_{i} \in Q_{i}$ we have

$$
\begin{aligned}
A_{i}^{-1}\left(y_{i}\right) & =\left\{\left[\left(\operatorname{co}\left(P_{i}\right)\right)^{-1}\left(y_{i}\right) \cap F_{i}^{-1}\left(y_{i}\right)\right] \cap N_{i}\right\} \cup\left[F_{i}^{-1}\left(y_{i}\right) \cap M_{i}\right] \\
& =\left[\left(\operatorname{co}\left(P_{i}\right)\right)^{-1}\left(y_{i}\right) \cap F_{i}^{-1}\left(y_{i}\right)\right] \cup\left[F_{i}^{-1}\left(y_{i}\right) \cap M_{i}\right] \\
& =\left[\left(\operatorname{co}\left(P_{i}\right)\right)^{-1}\left(y_{i}\right) \cup M_{i}\right] \cap F_{i}^{-1}\left(y_{i}\right)
\end{aligned}
$$


which is open in $Q$ by (2.5). Thus $B_{i} \in D K T\left(Q, Q_{i}\right)$. In addition (2.6) implies that $B_{i}$ is a compact map. Now Theorem 2.1 implies that there exists $x^{\star} \in Q$ with $x_{i}^{\star} \in B_{i}\left(x^{\star}\right)$ for all $i \in I$ (here $x_{i}^{\star}$ is the projection of $x^{\star}$ on $E_{i}$ ). Note if $i \in I\left(x^{\star}\right)$ for some $i \in I$, then $F_{i}\left(x^{\star}\right) \cap P_{i}\left(x^{\star}\right) \neq \emptyset$ and $x_{i}^{\star} \in \operatorname{co}\left(P_{i}\left(x^{\star}\right)\right) \cap G_{i}\left(x^{\star}\right)$ (in particular $x_{i}^{\star} \in \operatorname{co}\left(P_{i}\left(x^{\star}\right)\right)$ ). This contradicts (2.7). Thus $i \notin I\left(x^{\star}\right)$ for all $i \in I$. Consequently $F_{i}\left(x^{\star}\right) \cap P_{i}\left(x^{\star}\right)=\emptyset$ and $x_{i}^{\star} \in G_{i}\left(x^{\star}\right)$ for all $i \in I$.

THEOREM 2.6. Let $\Gamma=\left(Q_{i}, F_{i}, G_{i}, P_{i}\right)_{i \in I}$ be an abstract economy (here $I$ is countable) such that for each $i \in I$ the following conditions hold: $\left\{\begin{array}{l}Q_{i} \text { is a nonempty, closed, convex set in a metrisable } \\ \text { locally convex linear topological space } E_{i}\end{array}\right.$

$$
0 \in Q=\prod_{i \in I} Q_{i}
$$

$$
\text { for each } x \in Q, \quad F_{i}(x) \neq \emptyset \text { and } \operatorname{co}\left(F_{i}(x)\right) \subseteq G_{i}(x)
$$

$$
\left\{\begin{array}{l}
\text { for each } y_{i} \in E_{i}, \text { the set }\left[\left(\operatorname{co}\left(P_{i}\right)\right)^{-1}\left(y_{i}\right) \cup M_{i}\right] \cap F_{i}^{-1}\left(y_{i}\right) \\
\text { is open in } Q ; \text { here } M_{i}=\left\{x \in Q: F_{i}(x) \cap P_{i}(x)=\emptyset\right\}
\end{array}\right.
$$

$$
G_{i}: Q \rightarrow 2^{E_{i}} \text { is a compact map }
$$

and

(2.13) for each $x \in Q, x_{i} \notin c o\left(P_{i}(x)\right)$; here $x_{i}$ is the projection of $x$ on $E_{i}$.

Let $I(x)=\left\{i \in I: F_{i}(x) \cap P_{i}(x) \neq \emptyset\right\}$ and $B_{i}: Q \rightarrow 2^{E_{i}}$ be

$$
B_{i}(x)=\left\{\begin{array}{l}
\operatorname{co} P_{i}(x) \cap G_{i}(x) \text { if } i \in I(x) \\
G_{i}(x) \text { if } i \notin I(x) .
\end{array}\right.
$$

Define $B: Q \rightarrow 2^{E}$ (here $E=\prod_{i \in I} E_{i}$ ) by

$$
B(x)=\prod_{i \in I} B_{i}(x) \text { for each } x \in Q
$$

Assume

$$
\left\{\begin{array}{l}
\text { if }\left\{\left(x_{j}, \lambda_{j}\right)\right\}_{1}^{\infty} \text { is a sequence in } \partial Q \times[0,1] \text { converging to }(x, \lambda) \\
\text { with } x \in \lambda B(x) \text { and } 0 \leqslant \lambda<1 \text {, then there exists } j_{0} \in\{1,2, \ldots\} \\
\text { with }\left\{\lambda_{j} B\left(x_{j}\right)\right\} \subseteq Q \text { for each } j \geqslant j_{0}
\end{array}\right.
$$

holds. Then $\Gamma$ has an equilibrium. 
Proof: For each $i \in I$ let $N_{i}, A_{i}$ be as in Theorem 2.5. Essentially the same reasoning as in Theorem 2.5 implies that $B_{i} \in D K T\left(Q, E_{i}\right)$ is a compact map. Now Theorem 2.3 implies that there exists $x^{\star} \in Q$ with $x_{i}^{\star} \in B_{i}\left(x^{\star}\right)$ for all $i \in I$. The conclusion follows as in Theorem 2.5.

We conclude the paper by extending the main result in [9] if the spaces $E_{\boldsymbol{i}}$ are Fréchet (that is, complete, metrisable locally convex linear topological space). In the analysis we shall need the following result of the author $[6,7]$.

THEOREM 2.7. Let $E$ be a Fréchet space with $Q$ a closed, convex subset of $E$ and $0 \in Q$. Assume $G: Q \rightarrow C K(E)$ is a upper semicontinuous, compact map; here $C K(E)$ denotes the family of nonempty, convex, compact subsets of $E$. In addition suppose

$$
\left\{\begin{array}{l}
\text { if }\left\{\left(x_{j}, \lambda_{j}\right)\right\}_{1}^{\infty} \text { is a sequence in } \partial Q \times[0,1] \text { converging to }(x, \lambda) \\
\text { with } x \in \lambda G(x) \text { and } 0 \leqslant \lambda<1, \text { then there exists } j_{0} \in\{1,2, \ldots\} \\
\text { with }\left\{\lambda_{j} G\left(x_{j}\right)\right\} \subseteq Q \text { for each } j \geqslant j_{0}
\end{array}\right.
$$

holds. Then $G$ has a fixed point in $Q$.

REMARK. In fact Theorem 2.7 holds true if $E$ is just a metrisable, locally convex linear topological space (follow the ideas in $[6,7]$ using the fixed point theorem of Himmelberg for compact multifunctions).

We shall now use Theorem 2.7 together with some of the ideas in [9] to establish the following theorem.

Theorem 2.8. Let $\Gamma=\left(Q_{i}, F_{i}, G_{i}, P_{i}\right)_{i \in I}$ be an abstract economy (here $I$ is countable) such that for each $i \in I$ the following conditions hold: $Q_{i}$ is a nonempty, closed, convex set in a Fréchet space $E_{i}$

$$
\begin{gathered}
0 \in Q=\prod_{i \in I} Q_{i} \\
\left\{\begin{array}{l}
\text { for each } x \in Q, \quad F_{i}(x) \neq \emptyset \text { with } F_{i}(x) \subseteq G_{i}(x) \\
\text { and } G_{i}(x) \text { is convex }
\end{array}\right. \\
\left\{\begin{array}{l}
\text { the correspondence } \overline{G_{i}}: Q \rightarrow 2^{E_{i}} \text { defined by } \\
\overline{G_{i}}(x)=c_{E_{i}} G_{i}(x) \text { is upper semicontinuous }
\end{array}\right. \\
\text { for each } y_{i} \in E_{i}, F_{i}^{-1}\left(y_{i}\right) \text { is open in } Q \\
\text { for each } y_{i} \in E_{i}, P_{i}^{-1}\left(y_{i}\right) \text { is open in } Q \\
\overline{G_{i}}: Q \rightarrow 2^{E_{i}} \text { is a compact map }
\end{gathered}
$$

and

(2.23) for each $x \in Q, x_{i} \notin c o\left(P_{i}(x)\right)$; here $x_{i}$ is the projection of $x$ on $E_{i}$. 
Now for each $i \in I$ let

$$
\phi_{i}(x)=F_{i}(x) \cap \operatorname{co}\left(P_{i}(x)\right) \text { for } x \in Q \text { and } U_{i}=\left\{x \in Q: \phi_{i}(x) \neq \emptyset\right\}
$$

and

$$
H_{i}(x)=\left\{\begin{array}{l}
\left\{f_{i}(x)\right\} \quad \text { if } x \in U_{i} \\
\overline{G_{i}}(x) \text { if } x \notin U_{i}
\end{array}\right.
$$

where $f_{i}: U_{i} \rightarrow 2^{E_{i}}$ is a continuous selection of $\left.\phi_{i}\right|_{U_{i}}$ (see the proof for the existence of $f_{i}$ ). Define the map $H: Q \rightarrow 2^{E}$ (here $E=\prod_{i \in I} E_{i}$ ) by

$$
H(x)=\prod_{i \in I} H_{i}(x) \text { for each } x \in Q
$$

Assume

$$
\left\{\begin{array}{l}
\text { if }\left\{\left(x_{j}, \lambda_{j}\right)\right\}_{1}^{\infty} \text { is a sequence in } \partial Q \times[0,1] \text { converging to }(x, \lambda) \\
\text { with } x \in \lambda H(x) \text { and } 0 \leqslant \lambda<1, \text { then there exists } j_{0} \in\{1,2, \ldots\} \\
\text { with }\left\{\lambda_{j} H\left(x_{j}\right)\right\} \subseteq Q \text { for each } j \geqslant j_{0}
\end{array}\right.
$$

holds. Then $\Gamma$ has an equilibrium $x^{\star} \in Q$, that is, for each $i \in I$,

$$
x_{i}^{\star} \in \overline{G_{i}}\left(x^{\star}\right) \text { and } F_{i}\left(x^{\star}\right) \cap P_{i}\left(x^{\star}\right)=\emptyset ;
$$

here $x_{i}^{\star}$ is the projection of $x^{\star}$ on $E_{i}$.

REMARK. Theorem 2.8 remains valid if for each $i \in I, E_{i}$ is just a metrisable, locally convex linear topological space.

REMARK If $G_{i}(Q) \subseteq Q_{i}$ for each $i \in I$ then (2.24) is clearly satisfied. Consequently in [9, Theorem 6.1] is a special case of Theorem 2.8 .

REMARK More generally if $\bar{G}_{i}(\partial Q) \subseteq Q_{i}$ for each $i \in I$ then (2.24) is automatically true since $0 \in Q$ and

$$
H(\partial Q)=\prod_{i \in I} H_{i}(\partial Q) \subseteq \prod_{i \in I} Q_{i}=Q
$$

Proof: Fix $i \in I$. Let $\phi_{i}: Q \rightarrow 2^{E_{i}}$ and $U_{i}$ be as in the statement of Theorem 2.8 . Note (2.20), (2.21) and [9, Lemma 5.1] implies for each $y \in E_{i}$ that $\phi_{i}^{-1}(y)$ is open in $Q$. Also notice it is easy to see that

$$
U_{i}=\bigcup_{y \in E_{i}} \phi_{i}^{-1}(y)
$$

and so $U_{i}$ is open in $Q$. Now $E=\prod_{i \in I} E_{i}$ is metrisable and $U_{i}$ is paracompact. In addition we have (see the definitions of $\phi_{i}$ and $U_{i}$ ) that

$$
\psi_{i}=\left.\phi_{i}\right|_{U_{i}}: U_{i} \rightarrow 2^{E_{i}}
$$


has nonempty, convex values and for each $y \in E_{i}$,

$$
\psi_{i}^{-1}(y)=\left(\phi_{i}\right)^{-1}(y) \cap U_{i}
$$

is open in $U_{i}$. Now [9, Theorem 3.1] guarantees that there exists a continuous selection $f_{i}: U_{i} \rightarrow 2^{E_{i}}$ such that $f_{i}(x) \in \psi_{i}(x)$ for all $x \in U_{i}$. Let $H_{i}$ be as described in the statement of Theorem 2.8. Notice (2.19) together with [9, Lemma 6.1] implies that $H_{i}: Q \rightarrow 2^{E_{i}}$ is upper semicontinuous Also from (2.18) it is easy to see for each $x \in Q$ that $H_{i}(x)$ is a nonempty, closed and convex subset of $E_{i}$. Moreover (2.22) implies $H_{i}: Q \rightarrow C K\left(E_{i}\right)$ is a upper semicontinuous, compact map. Define $H$ as in the statement of Theorem 2.8. Clearly $H: Q \rightarrow C K(E)$ is a upper semicontinuous (see [1, p.472]), compact map. Now Theorem 2.8 implies that there exists $x^{\star} \in Q$ with $x^{\star} \in H\left(x^{\star}\right)$. If $x^{\star} \in U_{i}$ for some $i \in I$ then $x_{i}^{\star}=f_{i}\left(x^{\star}\right) \in F_{i}\left(x^{\star}\right) \cap \operatorname{co}\left(P_{i}\left(x^{\star}\right)\right) \subseteq \operatorname{co}\left(P_{i}\left(x^{\star}\right)\right)$ (here $x_{i}^{\star}$ is the projection of $x^{\star}$ on $E_{i}$ ). This contradicts (2.23). Thus for each $i \in I$ we must have $x^{\star} \notin U_{i}$ so that $x_{i}^{\star} \in \overline{G_{i}}\left(x_{i}\right)$ and $F_{i}\left(x^{\star}\right) \cap \operatorname{co}\left(P_{i}\left(x^{\star}\right)\right)=\emptyset$.

\section{REFERENCES}

[1] C.D. Aliprantis and K.C. Border, Infinite dimensional analysis, (Springer Verlag, Berlin, Heidelberg, New York, 1991).

[2] X.P. Ding, W.K. Kim and K.K. Tan, 'A selection theorem and its applications', Bull. Austral. Math. Soc. 46 (1992), 205-212.

[3] J.Dugundji and A. Granas, Fixed point theory, Monografie Matematyczne (PWN, Warsaw, 1982).

[4] P.M. Fitzpatrick and W.V. Petryshyn, 'Fixed point theorems for multivalued noncompact acyclic mappings', Pacific J. Math. 54 (1974), 17-23.

[5] D. O'Regan, 'Some fixed point theorems for concentrative mappings between locally convex linear topological spaces', Nonlinear Anal. 27 (1996), 1437-1446.

[6] D. O'Regan, 'Fixed point theory for compact upper semi-continuous or lower semi-continuous set valued maps', Proc. Amer. Math. Soc. 125 (1997), 875-881.

[7] D. O'Regan, 'Fixed points for set valued mappings in locally convex linear topological spaces', Math. Comput. Modelling (to appear).

[8] E. Tarafdar, 'A fixed point theorem and equilibrium point of an abstract economy', $J$. Math. Econom. 20 (1991), 211-218.

[9] N. Yannelis and N. Prabhaker, 'Existence of minimal elements and equilibria in linear topological spaces,', J. Math. Econom. 12 (1983), 233-246.

Department of Mathematics

Nationional University of Ireland

Galway

Ireland 